\title{
Pruned Volterra Models with Memory Effects for Nonlinear Power Amplifiers
}

\author{
Pengpeng Li ${ }^{1}$, Qingfang Zhang ${ }^{1}$, Ping Wang ${ }^{1}$, Zhongshan Xie ${ }^{2}$, Bing Liu ${ }^{2}$ \\ ${ }^{1}$ Shanghai Institute of Microsystem and Information Technology, Shanghai, China \\ ${ }^{2}$ Nanjing University of Aeronautics and Astronautics, Nanjing, China \\ Email: pingwang@mail.sim.ac.cn, zhongshanxie@hotmail.com
}

Received May 2013

\begin{abstract}
In this letter, a novel model is proposed for modeling the nonlinearity and memory effects of power amplifiers. The classical Volterra model is modified through a function of the sum of nonlinearity order with sum of memory length. The parameters of this model can be extracted in digital domain since the model is analyzed based on the envelope signals. The model we proposed enables a substantial reduction in the number of coefficients involved, and with excellent accuracy.
\end{abstract}

Keywords: Volterra Series; Power Amplifier (PA); Behavioral Model; Memory Effect

\section{Introduction}

To handle multi-carrier envelope varying signals with wide bandwidths in modern wireless communication system, the signals passing through the transistor and power amplifiers should be able to predict accurately. In the behavioral model, the nonlinear component is generally considered as a "black-box" which is completely characterized by external responses, in terms of input and output signals, through the use of relatively simple mathematical expressions. The Volterra series is a general nonlinear model with memory and has been used by many researchers to characterize power amplifiers [1,2]. Unfortunately, the number of coefficients of the Volterrabased models becomes unacceptable in practical implementation as the accuracy level increased, such models become useless.

To overcome the modeling complexity, various model-order reduction approaches have been proposed to simplify the Volterra model structure. For example, Wiener, Hammerstein, Wiener-Hammerstein [3,4] and Memory Polynomial [5] models are the most popular approximations. Physical knowledge has been considered in recently proposed models by taking into account the real behavior of the PA [6]. And the device electrical properties were also considered in the reduction [7].

Although these simplified models have been employed to characterize PAs with reasonable accuracy under certain conditions, the number of coefficients to be estimated is still increasing prominently with the degree of nonlinearity and memory length of the system. While the interaction between the nth order and memory length has not attracted enough attention.

The model proposed in this work is based on an underlying general Volterra model of the PA. By focusing on the fact that output items is fading with the non-linearity order and memory length increase, the Volterra model is modified. As the function of nonlinearity and memory effects applied, a slow growth of the coefficients can be obtained while the accuracy can be improved. The letter is organized as follows: after this introduction, Volterra model is modified based on the nonlinearity and memory effects analyzed in Section 2. The model's performance is shown in Section 3. At last the conclusion is presented in Section 4.

\section{Principle of Proposed Model}

A Volterra series is a combination of linear convolution and a nonlinear power series so that it can be used to describe the input/output relationship of a general nonlinear, causal and time-invariant system with fading memory.

$y(n)=\sum_{p=1}^{p} \sum_{i_{1}=0}^{\prime \prime} \ldots \sum_{i_{p}=0}^{\prime \prime} h_{p}\left(i_{1}, \ldots, i_{p}\right) \prod_{j=1}^{p} x\left(n-i_{j}\right)$

where $x(n)$ and $y(n)$ represents the input and the output, $\mathrm{P}$ and $\mathrm{M}$ are the order of nonlinearities and the memory length, respectively, and $h_{p}\left(i_{1}, \ldots, i_{p}\right)$ is the discrete time Volterra kernels of order $n$.

In the application of wide-band system, memory ef- 
fects of the amplifier are very significant and have an important impact on linear effects. Memory effects [8] can be classified into electrical and thermal. The electrical memory effects arise as a consequence of the variation of the impedance along the signal bandwidth modulation.

As the output of the power amplifier nonlinearity will vanish at the infinite order and the memory effects will fade out with time passing by, there must be an exponential role in the amplitude function.

To make it easy to understand the proposed model, first we consider the output of the memoryless system is a function of nonlinearity order $p$, which can be expressed as follows:

$$
y(n)=\sum_{p=1}^{p} y_{p}(n)=\sum_{p=1}^{p} f(p) X^{p}(n)
$$

When the polynomial contains memory, the change of the amplitude must be the function of memory which can be written as

$$
y_{p}(n-m)=f(p) g(m)_{X}{ }^{p}(n-m)
$$

According to Equation (3), the function of memory $g(m)$ can be written as

$$
g(m)=\frac{y_{p}(n-m)}{f(p)_{X}{ }^{p}(n-m)}
$$

When we consider the error of the polynomial, if $\left\|\operatorname{Max}\left(y_{p}(n-m)\right)\right\|<\varepsilon$, the polynomial will be replaced by $\varepsilon$ ( $\varepsilon$ can be calculated by the value of IMD and gain of the PA). Obviously the output can get the maximum value when the input takes the maximum value, so we can get the function of the memory with the nonlinearity order $p$ as following (the input has been normalized):

$$
m(p) \leq g^{-1}\left(\left\|\frac{\varepsilon}{f(p)}\right\|\right)
$$

Considering the memory effects of PAs, the function of memory $g(m)$ would fade gradually, as $g(0)=1, g(\infty)$ $=0$, we can assume $g(n)=e^{-\alpha n}$ and that corresponds with the characteristic of capacitance or inductive components which can release the energy, then Equation (5) can be written as

$$
m(p) \leq-\frac{1}{\alpha} \ln \left(\left\|\frac{\varepsilon}{f(p)}\right\|\right)
$$

And the multiply items such as $x^{p_{1}}\left(n-m_{1}\right) X^{p_{2}}\left(n-m_{2}\right)$ can be written as

$$
\sum m \leq-\frac{1}{\alpha} \ln \left(\left\|\frac{\varepsilon}{\prod f(p)}\right\|\right)
$$

where $f(p)$ is a function which depends on the nonlinearity of the PAs and decreased as the nonlinearity order increased. To simplify the analyzing, we can assume $f(p)=e^{-\beta p}$ (has been normalized as same as input), then last-written equation can be written as

$$
\alpha \sum m+\beta \sum p \leq-\ln \varepsilon
$$

To attain the value of $\beta$ and $\varepsilon$, the input power, IMD and gain of the PA must be known. For example, assuming the parameters of a definite PA are $P_{i n}=P_{0}$, IMD5 $=I_{5}$ and gain $=\mathrm{G}$. From the definition of IMD5, $I_{5}$ can be written as

$$
I_{5}=10 \log \frac{P_{5}}{P_{i n}}
$$

as $P_{5}=\frac{1}{2} \frac{y_{5}^{2}}{50}$ and $\left|y_{5}\right|_{\max }=\varepsilon \times G$, so $\varepsilon$ can be determined by

$$
\varepsilon=\frac{\sqrt{P_{0} \times 10^{-I_{5} / 20}}}{G}
$$

Take (10) into (8), since $\sum p=5$, then the $\beta$ can be determined by

$$
\beta=-\frac{1}{5} \ln \frac{\sqrt{P_{0} \times 10^{-I_{5} / 20}}}{G}
$$

Note that $\alpha$ is the only unknown variable in (8) and $\alpha$ depends on the memory effects of PA. However, the memory effects of PA cannot be tested, so we attain a relative accurate $\alpha$ through comparing model performance of different $\alpha$.

Evidently the function of $\sum m$ and $p$ is a decreasing function so that the coefficients can be decreased rapidly when the system's order increased.

\section{Maintaining the Integrity of the Specifications}

The template is used to format your paper and style the text. All margins, column widths, line spaces, and text fonts are prescribed; please do not alter them. You may note peculiarities. For example, the head margin in this template measures proportionately more than is customary. This measurement and others are deliberate, using specifications that anticipate your paper as one part of the entire proceedings, and not as an independent document. Please do not revise any of the current designations.

\section{Model Performance}

In order to validate the proposed behavioral model in a real system, a Doherty PA was tested. This PA was operated at $460 \mathrm{MHz}$ and excited by an OFDM signal with 20 MHz bandwidth.

The parameters of tested PA were Pin $=10 \mathrm{~W}$, IMD5 $=-40 \mathrm{dBc}$ and gain $=20 \mathrm{~dB}$. So the $\varepsilon$ and $\beta$ can be fixed by (10) and (11). Thus (8) can be written as

$$
\alpha \sum m+0.8 \sum p \leq 4.2
$$


In this test, the nonlinearity of the model was truncated to order 5. For comparison, the value of $\alpha$ was set from 0.2 to 2 . To evaluate the model's fidelity in the time domain, the NMSEs and number of coefficients for each partial model were calculated. These results are shown in Figure 1. Due to the fact that memory effects of different PAs were not the same, so the value of $\alpha$ was different for different PAs. In this paper, the value of $\alpha$ was set to 1.6 while the performance of proposed model for the tested PA was better and with lesser number of coefficients.

To show the model accuracy in the frequency domain, the spectra of modeled errors are plotted in Figure 2. It can be seen that the error signal spectrum of our proposed model is very small, while significant errors are generated in the output predicted by the memoryless model. For reference, the spectrum of the simulated output is also plotted in Figure 2.

\section{Conclusion}

An efficient and effective Volterra model pruning me-

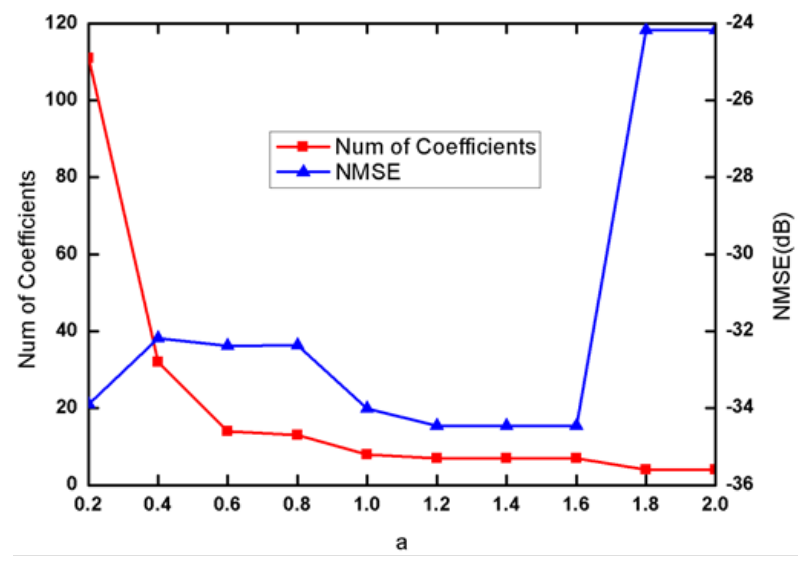

Figure 1. Model performance in the time domain.

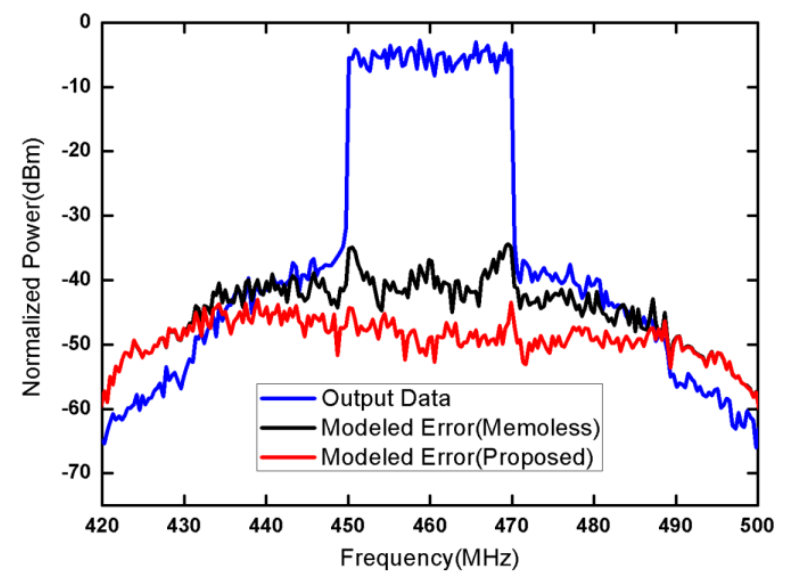

Figure 2. Sample frequency domain output and modeled error spectra. thod for RF PAs has been presented in this letter, which based on a function of the sum of nonlinearity order with the sum of memory length. The advantage of this model reduction approach is that it allows efficient reduction of the model complexity, while keeping the essential properties caused by memory effects of a real PA. With a Doherty PA tested, the proposed model can be employed to characterize a nonlinear PA with memory effects in high accuracy.

\section{Acknowledgements}

The research work is supported by Chinese Major National Science and Technology Projects

(No.2010ZX03007-003).

\section{REFERENCES}

[1] M. Schetzen, "The Volterra and Wiener Theories of Nonlinear Systems,” R. E. Krieger Publishing, Malabar, 1989.

[2] V. J. Mathews and G. L. Sicuranza, "Polynomial Signal Processing,” John Wiley \& Sons, New York, 2000.

[3] C. P. Silva, et al, "Optimal-filter Approach for Nonlinear Power Amplifier Modeling and Equalization”, IEEE MTT-S International Microwave Symposium Digest, Boston, June 2000, pp. 437-440.

[4] H. Ku, M. Mckinley and J. S. Kenney, "Quantifying Memory Effects in RF Power Amplifiers," IEEE Transactions on Microw Theory and Tech, Vol. 50, No. 12, 2002, pp. 2843-2849. http://dx.doi.org/10.1109/TMTT.2002.805196

[5] J. Kim and K. Konstantinou, "Digital Predistortion of Wideband Signals Based on Power Amplifier Model with Memory,” Electronics Letters, Vol. 37, No. 23, 2001, pp. 1417-1418. http://dx.doi.org/10.1049/el:20010940

[6] A. Zhu, J. C. Pedro and T. R. Cunha, "Pruning the Volterra Series for Behavioral Modeling of Power Amplifiers Using Physical Knowledge," IEEE Transactions on Microw Theory and Tech, Vol. 55, No. 5, 2007, pp. 813821.

[7] C. Crespo-Cadenas, J. Reina-Tosina and M. J. MaderoAyora, "Volterra Behavioral Model for Wideband RF Amplifiers," IEEE Transactions on Microw Theory and Tech, Vol. 55, No. 5, 2007, pp. 449-457.

[8] J. Vuolevi, T. Rahkonen and J. Manninen, "Measurement Technique for Characterizing Memory Effects in RF Power Amplifiers," IEEE Transactions on Microw Theory and Tech, Vol. 49, 2001, pp. 1383-1389.

http://dx.doi.org/10.1109/22.939917 